\title{
O DESENVOLVIMENTO MOTOR E A INFLUENNCIA DO AMBIENTE FAMILIAR E DO NÍVEL SOCIOECONÔMICO
}

Larissa Wagner Zanella, Universidade Federal do Rio Grande do Sul - UFRGS, Porto Alegre, Rio Grande do Sul-Brasil

Carla dos Reis Rezer, Universidade Comunitária da Região de Chapecó UNOCHAPECO, Chapecó, Santa Catarina-Brasil

\section{RESUMO}

O termo desenvolvimento motor é empregado em estudos que pretendem compreender os processos de desenvolvimento da motricidade humana. $\mathrm{O}$ processo de desenvolvimento motor ocorre de acordo padrões biológicos também pela influência de fatores externos. $\mathrm{O}$ objetivo deste estudo desta revisão foi rever considerações importantes acerca do desenvolvimento motor baseados na obra de Gallahue, Ozmun e Goodway e verificar a influência do ambiente e do nível socioeconômico sobre o desenvolvimento motor de crianças. Foram investigados estudos coletados no período de setembro de 2013 a fevereiro de 2014 nas bases de dados Google Acadêmico, Scielo e Ebsco. A revisão dos estudos reforça que o ambiente familiar e o nível socioeconômico podem influenciar no desenvolvimento motor infantil.

Palavras-Chave: Desenvolvimento motor; Ambiente familiar; Nível socioeconômico.

\section{MOTOR DEVELOPMENT AND INFLUENCE OF FAMILY ENVIRONMENT AND SOCIO-ECONOMIC LEVEL}

\begin{abstract}
The term motor development is employed in studies that seek to understand the development processes of human movement. The process of motor development occurs according biological standards also by the influence of external factors. The aim of this study was to review this important considerations about motor development based on the work of Gallahue, Ozmun and Goodway and the influence of environment and socioeconomic status on motor development of children. Collected studies were investigated in period September 2013 to February 2014 on the basis of Google Academic, Scielo and Ebsco. A review of studies reinforces the family environment and socioeconomic status can influence the infant motor development.
\end{abstract}

Key-Words: Motor development; Home environment; Socioeconomic status. 


\title{
DESARROLLO MOTOR Y LA INFLUENCIA DEL ENTORNO FAMILIAR Y NIVEL SOCIOECONÓMICO
}

\begin{abstract}
RESUMEN
El desarrollo motor término se emplea en los estudios que tratan de entender los procesos de desarrollo del movimiento humano. El proceso de desarrollo motor se produce según normas biológicas también por la influencia de factores externos. El objetivo de este estudio fue revisar la opinión Este importantes consideraciones sobre el desarrollo motor basado en el trabajo de Gallahue, Ozmun y Goodway y la influencia del medio ambiente y la situación socioeconómica en el desarrollo motor de los niños. Se investigaron los estudios recogidos en el período septiembre 2013-febrero 2014 sobre la base de Google Scholar, Scielo y Ebsco. La revisión de estudios refuerza el entorno familiar y la situación socioeconómica puede influir en el desarrollo motor infantil.
\end{abstract}

Palabras-Clave: Desarrollo motor; Ambiente familiar; Estatus socioeconómico. 


\section{INTRODUÇÃO}

O termo desenvolvimento motor é empregado em estudos que pretendem compreender os processos de desenvolvimento da motricidade humana. ${ }^{1-2}$ Pesquisadores $^{3-8}$ tem se dedicado à investigar as mudanças no comportamento motor e os fatores que subjazem a essas mudanças. Compreender as primeiras tentativas em locomover-se: os primeiros passos, em diversas direções, com equilíbrio e segurança, e, então, saltar e correr com independência no ambiente em que vive, pode tornar-se um desafio extremamente motivante. A infância é considerada a fase ideal para o desenvolvimento de uma gama de habilidades motoras fundamentais. ${ }^{1}$ A aquisição destas habilidades motoras possibilitará que a criança desenvolva um amplo domínio do seu corpo em posturas diferenciadas de equilíbrio, locomoção e controle de objetos. ${ }^{1}$ Ainda mais, tais habilidades são essenciais para a condução de rotinas básicas diárias e para o engajamento em atividades esportivas de lazer e/ou de competição.

O desenvolvimento motor sofre influências do crescimento e características maturacionais da criança (morfológica, fisiológica e neuromuscular). À medida que a motricidade é desenvolvida em um contexto social específico, o ambiente em que a criança está inserida também torna-se um fator de influência importante e que deve ser investigado. ${ }^{9-12}$ Contextos diferenciados oferecem demandas específicas sobre as competências motoras e atividades físicas de bebês e crianças. $^{11} \mathrm{O}$ ambiente familiar $^{13,14}$ e $\mathrm{o}$ nível socioeconômico $^{12}$ são fatores potencialmente importantes e que devem ser levados em consideração. Portanto, o objetivo desta revisão foi rever considerações importantes acerca do desenvolvimento motor baseada na obra de Gallahue, Ozmun e Goodway ${ }^{1}$ e verificar a influência dos fatores citados sobre o desenvolvimento motor das crianças.

Este artigo compreende dois momentos, o primeiro momento é direcionado para a revisão de conteúdo através da obra de Gallahue, Ozmun e Goodway. ${ }^{1}$ A segunda parte faz uma análise das possíveis influências da família e do nível socioeconômico no desenvolvimento motor de crianças.

A pesquisa por artigos científicos ocorreu nas seguintes bases de dados: Google Acadêmico, EBSCO e SCIELO no período de setembro de 2013 até fevereiro de 2014. Na busca não foi delimitado um período de abrangência das publicações, ou seja, todos os 
artigos publicados até o ano de 2014 e disponíveis nas bases de dados foram investigados. Os artigos coletados neste período foram avaliados por título, resumo, metodologia segundo os critérios de inclusão e exclusão estabelecidos previamente. Os critérios para a inclusão de artigos foram (1) idade da amostra (0 a 10 anos); (2) investigar o desenvolvimento motor, nível socioeconômico e/ou ambiente familiar; (3) texto publicado integralmente. Não foram incluídos artigos cuja amostra era diagnosticada com desordens, transtornos, deficiências motoras e/ou cognitivas. Os termos utilizados para a busca de artigos foram "desenvolvimento motor", "fatores de influência", "ambiente familiar" e "nível socioeconômico" isolados ou combinados. A busca de artigos foi realizada com tais termos em idioma português e inglês e resultou em 104 artigos. Após a análise 14 artigos obedeceram aos critérios de inclusão e compuseram esta revisão.

\section{UMA BREVE REVISÃO SOBRE DESENVOLVIMENTO MOTOR}

O estudo do desenvolvimento motor pretende basicamente descrever e explicar as modificações no comportamento motor humano ao longo da vida. Um objetivo tão abrangente recorre necessariamente a contributos de diferentes áreas do conhecimento, especialmente as tradicionalmente ligadas ao estudo da evolução de organismos vivos, numa perspectiva biológica, ou as que se preocupam com o estudo do comportamento humano considerado individual ou social. Esses comportamentos e interações incluem aspectos fundamentais como a nutrição, organização de hábitos, modelação de comportamentos por intervenção educativa ou simplesmente por imitação.

Nos primeiros anos de vida os cuidados maternos são decisivos. Aos poucos essa responsabilidade de estimulação vai sendo incorporada, outros e novos agentes aumentam as fontes de interferência no desenvolvimento da criança. Lentamente o organismo tornarse-á apto para realizar movimentos de maior complexidade. A complexidade dos movimentos não pode ser observada na primeira infância, entretanto, por volta dos dois anos de vida é possível identificar um repertório motor rudimentar que inclui movimentos e habilidades necessárias à maioria das tarefas do quotidiano. Praticamente todos os movimentos fundamentais do comportamento motor de um adulto podem ser observados no final da primeira infância. Isso inclui os movimentos mais exigentes em termos de precisão e minúcia de movimento, como a preensão, por exemplo, até habilidades de locomoção ou organização postural.

Conexões: revista da Faculdade de Educação Física da UNICAMP, Campinas, v. 13, n. 3, p. 101-113, jul./set. 2015. ISSN: 1983-9030 
Nessa fase, a criança torna-se mais forte, resistente e coordenada, possibilitando uma modificação quantitativa e qualitativa das atividades físicas. Isto irá refletir na natureza das brincadeiras preferidas pela criança e, sobretudo na manifestação de uma tendência para a especialização-individualização do seu comportamento futuramente. Esta fase de transição corresponde, à grosso modo, ao período do salto pubertário durante o qual irão ocorrer profundas transformações hormonais e morfológicas, mas também motoras e comportamentais. É na adolescência que, geralmente, ocorre a especialização dos movimentos, restringindo e simultaneamente aperfeiçoando as opções motoras individuais.

O crescimento prolonga-se até a idade adulta e a busca de uma maior eficiência corporal continua por muitos anos mais. Inevitavelmente, o ciclo da vida obrigará a uma progressiva redução das possibilidades corporais, acompanhada de alterações estruturais e funcionais de intensidade e extensão variáveis. Portanto, em cada ciclo de vida o movimento poderá tomar características significativas e a aquisição de determinados comportamentos motores pode ter repercussões importantes no desenvolvimento do indivíduo. Cada aquisição é influenciada pela anterior, tanto no domínio mental como no motor, através da experiência e troca com o meio.

\section{FASES DO DESENVOLVIMENTO MOTOR}

O ser humano está envolvido em um processo permanente de aprendizagem. É fundamental aprende a mover-se com controle e competência, em relação aos desafios enfrentados diariamente em um mundo que está em constante mudança. Podemos observar diferenças de desenvolvimento no comportamento motor provocadas por fatores próprios do indivíduo (biologia), do ambiente (experiência) e da tarefa em si (físicos/mecânicos). Esses fatores de alterações no comportamento motor podem ser observados através da forma e do desempenho motor. Uma forma adequada para analisar as alterações no comportamento motor é analisar o desempenho de acordo com os padrões motores. Os padrões motores são estabelecidos dentro de fases de desenvolvimento que são relativas ao comportamento observado ao longo de muitos estudos. As fases de desenvolvimento organizadas por Gallahue, Ozmun e Goodway ${ }^{1}$ contemplam a fase motora reflexiva, movimentos rudimentares, habilidades motoras fundamentais e movimentos especializados. 


\section{(I) Motora reflexiva}

A fase motora reflexiva contemplam os primeiros movimentos do bebê. Os reflexos são movimentos involuntários que formam a base para as fases posteriores do desenvolvimento motor. Os movimentos involuntários e a crescente sofisticação cortical nos primeiros meses de vida pós-natal desempenham papel para auxiliar a criança a aprender mais sobre o seu corpo e o mundo exterior. É através destes movimentos que a criança irá adquirir experiência e prática para desenvolver os movimentos da próxima fase.

Os reflexos primitivos e posturais compõem uma forma de movimentos involuntários e são aparentemente similares a comportamentos voluntários posteriores. A fase reflexiva do desenvolvimento motor pode ser dividida em dois estágios sobrepostos: (1) estágio de codificação de informações que é caracterizado por atividade motora involuntária observável no período fetal até aproximadamente o quarto mês do período pós-natal. Neste estágio, os movimentos ainda não são controlados e são incapazes de reunir informações, buscar alimento e encontrar proteção. (2) estágio de decodificação de informações inicia aproximadamente no quarto mês de vida. Nesse período, há gradual inibição de muitos reflexos a medida que os centros cerebrais superiores continuam a desenvolver-se. $\mathrm{O}$ estágio de codificação substitui a atividade sensório-motora por habilidade motorreceptiva. Isto é, o desenvolvimento do controle voluntário dos movimentos do bebê passa a envolver o processamento de estímulos sensoriais com informações armazenadas e ele não faz simplesmente a reação aos estímulos.

\section{(II) Movimentos rudimentares}

As primeiras formas de movimentos voluntários são os movimentos rudimentares, observados no bebê, desde o nascimento até, aproximadamente, a idade de dois anos. Os movimentos rudimentares são determinados de forma maturacional. Essa fase pode ser dividida em dois estágios: (1) estágio de inibição de reflexos se refere aos reflexos primitivos e posturais que são substituídos por comportamentos motores voluntários. Quanto à inibição de reflexos, o movimento voluntário é fragilmente diferenciado e integrado porque o aparato neuromotor do bebê está ainda em estágio rudimentar de desenvolvimento. (2) estágio pré-controle inicia por volta de um ano de idade onde as crianças começam a ter precisão e controle maiores sobre seus movimentos. Neste estágio, as crianças aprendem a obter e a manter seu equilíbrio, a manipular objetos e a locomover- 
se pelo ambiente com notável grau de proficiência e controle, considerando-se o curto período que tiveram para desenvolver essas habilidades.

\section{(III) Habilidades motoras fundamentais}

As habilidades motoras fundamentais da primeira infância são consequência da fase de movimentos rudimentares do período neonatal. Esta fase do desenvolvimento motor representa um período no qual as crianças pequenas estão ativamente envolvidas na exploração e na experimentação das capacidades motoras de seus corpos. Os padrões de movimento fundamentais são padrões básicos de comportamento observáveis. Habilidades locomotoras (corridas e saltos), habilidades manipulativas (arremessar, receber, rebater, rolar, quicar) e estabilizadoras (andar com firmeza, equilibrar-se em um pé só) são exemplos de habilidades fundamentais que devem iniciar nos dois primeiros anos da infância. Este estágio divide-se em três fases: (1) inicial representa as primeiras tentativas da criança orientadas para o objetivo de desempenhar uma habilidade motora fundamental; (2) emergente envolve maior controle e melhor coordenação rítmica das habilidades motoras fundamentais. Também é um estágio onde inicia a sincronização dos elementos temporais e espaciais do movimento. (3) proficiente é caracterizado por desempenhos motores eficientes, coordenados e controlados. O estudo do desenvolvimento motor de muitos anos sugere que as crianças devem atingir o estágio maduro no máximo até os sete anos de idade.

\section{(IV) Movimentos especializados}

Esta fase é resultado da fase das habilidades motoras fundamentais. $\mathrm{Na}$ fase especializada, o movimento torna-se uma ferramenta que se aplica a muitas atividades motoras complexas presentes na vida diária, na recreação e nos objetivos esportivos. A fase de movimentos especializados tem três estágios: (1) estágio transitório pode iniciar por volta dos oito ou nove anos de idade. A criança começa a combinar e a aplicar as habilidades motoras fundamentais ao desempenhar as habilidades especializadas no esporte e em ambientes recreacionais, por exemplo, chutar uma bola no jogo de futebol. As habilidades transitórias são simplesmente as aplicações das habilidades motoras fundamentais, de algum modo, em situações mais específicas e complexas. (2) estágio de aplicação ocorre aproximadamente entre onze e treze anos de idade. Neste estágio ocorrem mudanças interessantes e se referem à sofisticação cognitiva crescente e certa base ampliada de 
experiências que tornarão o pré-adolescente capaz de tomar numerosas decisões de aprendizado e de participação baseados em mitos da tarefa individuais e ambientais. Os indivíduos começam a buscar ou a evitar a participação em atividades específicas. (3) estágio de utilização permanente inicia por volta dos quatorze anos de idade e continua por toda a vida adulta. Esse estágio é caracterizado pelo uso do repertório de movimentos adquiridos pelo indivíduo por toda a vida. Em essência, o estágio de utilização permanente representa a cume de todos os estágios e fases precedentes. Ele deve, entretanto, ser considerado como uma continuação do processo permanente.

\section{FAMÍLIA E NÍVEL SOCIOECONÔMICO: FATORES DE INFLUÊNCIA NO DESENVOLVIMENTO MOTOR INFANTIL}

O ambiente familiar em que a criança está inserida normalmente é apontado como um fator importante de influência no desenvolvimento motor de uma criança. Alterações nos costumes e práticas na infância destacam o papel dos pais na criação de oportunidades e experiências motoras. Como na primeira infância, os pais promovem as oportunidades através da organização e/ou restrição de circunstâncias para o desenvolvimento de competências dos bebês. ${ }^{2}$ Os cuidadores decidem se as crianças ficarão no chão, ${ }^{3}$ se terão acesso a escadas ${ }^{4}$ ou onde irão dormir. ${ }^{5}$ A qualidade do ambiente familiar parece estar diretamente associada ao desenvolvimento motor das crianças, sendo os meninos mais suscetíveis do que as meninas para as influências que os cercam. ${ }^{4,6}$

Um indicativo da influência da família no desenvolvimento de crianças foram os resultados de um estudo com 28 crianças com idade entre 3 e 6 anos que haviam sido expostas a cocaína e outras drogas e cresceram com mães adotivas. ${ }^{7}$ Os atrasos no desenvolvimento motor observados durante a infância tinham sido superados no momento da avaliação. Os pesquisadores atribuíram às condições educativas favoráveis e à intervenção oportuna após o atraso motor o fato de as crianças apresentarem pontuações mais altas no desempenho motor.

Acredita-se que um ambiente permissivo da família contribui para o desenvolvimento

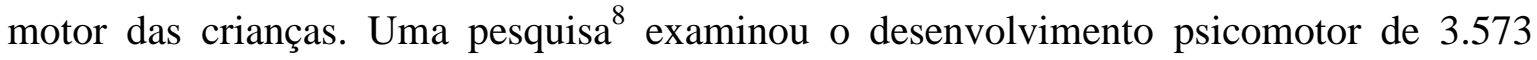
meninos e meninas, com idades 1 mês e 6 anos na Argentina. Os resultados indicaram que maior nível socioeconômico e nível de escolaridade da mãe foram relacionados com o 
melhor desempenho psicomotor em crianças ao longo do $1^{\circ}$ ano de idade. Os pesquisadores observaram que, as situações de baixo nível socioeconômico possuem correlação com as melhores pontuações nos testes de desenvolvimento durante a infância causados por práticas de educação de crianças indígenas que têm uma influência positiva sobre desenvolvimento, como cuidados de enfermagem e contato próximo entre bebê e sua mãe.

No entanto, a maioria dos estudos que investigaram amostras com crianças de classes sociais mais baixas reportam que estas apresentam um desempenho motor pior do que as crianças de classe média. ${ }^{9-12}$ É possível que problemas perceptuais-motores estejam relacionados à má nutrição pré e pós-natal, fato que pode afetar o sistema nervoso central. Isolando os aspectos relacionados à desnutrição e considerando apenas os aspectos motores, é possível que os baixos desempenhos motores apresentados por essas crianças podem ser simplesmente devidos à uma falta de experiência motora. Crianças que não foram incentivadas a desenvolver habilidades como a coordenação motora fina poderão apresentar dificuldades no desempenho escolar. À medida que, as crianças, de baixo a alto nível socioeconômico, residentes em zonas urbanas, especialmente aquelas que vivem em blocos de apartamentos, poderão sofrer com a falta de espaço adequado, fato que dificultará o processo de desenvolvimento das suas habilidades motoras fundamentais.

Entretanto, o que pode diferenciar o nível de desempenho motor das crianças com nível socioeconômico mais alto são as oportunidades de instrução adequada em ambientes esportivos (por exemplo, escolinhas ou clubes). Portanto, mesmo residindo em blocos de apartamentos, a falta de espaço é compensada por aulas extras de educação física. Além do mais, é possível que as crianças pertencentes às classes de nível socioeconômico mais alto podem contar um maior número e grande variedade de brinquedos em comparação as crianças de nível socioeconômico mais baixo. ${ }^{12}$ Por outro lado um estudo, ${ }^{16}$ embora antigo, reporta que o nível socioeconômico não afeta o desempenho motor das crianças britânicas até um ano de idade. No mesmo sentido, estudos desenvolvidos na Europa sugerem que a influência do nível socioeconômico da família no desenvolvimento da criança inicia a partir de um ano de idade. Por exemplo, um estudo ${ }^{11}$ desenvolvido com 2.309 crianças de 6 a 10 anos na Alemanha reporta que crianças com níveis socioeconômicos mais altos apresentam melhores níveis de desempenho motor. Os pesquisadores remetem essa 
vantagem à maior participação em esportes extraescolares que seus pares.

Estudos desenvolvidos no Brasil reportam a preocupação dos pesquisadores acerca dos fatores que podem influenciar nos atrasos motores em crianças de 0 a 48 meses. ${ }^{13-14}$ Uma pesquisa desenvolvida no nordeste do país ${ }^{13}$ investigou os ambientes familiares de crianças com idades entre 18 e 48 meses, através de entrevista com 128 tutores responsáveis pelas crianças. Os pesquisadores observaram prevalência da inadequação das residências e a inexistência de materiais suficientes ao desenvolvimento da motricidade grossa e fina de crianças Para amenizar problemas referentes à falta de estímulo familiar ou desigualdades sociais, o desenvolvimento de programas de intervenção motora precoce pode ser fundamental. Um estudo desenvolvido no sul do país ${ }^{14}$ implementou um programa de intervenção motora para bebês de 0 a 18 meses com a participação de pais e/ou cuidadores. As mães e cuidadoras do grupo interventivo recebiam orientações de atividades/tarefas motoras para bebês e de mudanças no contexto a cada semana durante um período de 8 semanas. Os resultados observados demonstraram importantes mudanças no desempenho motor dos bebês do grupo interventivo. Nesse sentido, é possível inferir que bebês, quando estimulados de maneira correta em ambiente familiar, podem desenvolver maior qualidade e controle de movimentos, o que poderá repercutir em benefícios motores em longo prazo.

\section{CONSIDERAÇÕES FINAIS}

O processo de desenvolvimento motor ocorre de acordo com o padrão que é estabelecido pela influência biológica. Além disso, o ambiente em que a criança está inserida pode determinar a gravidade do impacto das dificuldades. A modificação no ambiente pode determinar diferenças essenciais no progresso motor de uma criança. Com isso, a família e o nível socioeconômico são fatores que podem influenciar consideravelmente no percurso do desenvolvimento infantil. Então, embora as dificuldades motoras sejam consideradas condições permanentes, elas podem ser drasticamente melhoradas através da adoção de estratégias na família que contribuam para a estimulação motora. Além de oferecer oportunidades motoras adequadas através de programas interventivos para todas as crianças, principalmente para as crianças de nível socioeconômico mais baixo. 


\section{REFERÊNCIAS}

${ }^{1}$ GAllahuE, D. L.; OZMUN, J. C.; GOODWAY, J. Compreendendo o desenvolvimento motor: bebês, crianças, adolescentes e adultos. Porto Alegre: AMGH, 2013.

${ }^{2}$ REED, E. S.; BRIL, B. The primacy of action in development. In: BERNSTEIN, N. A. Dexterity and its development. Dinamarca: Lawrence Erlbaum Associates, 1996. p. 431451.

${ }^{3}$ ADOLPH, K. Learning to keep balance. Advances in child development and behavior, San Diego, v. 30, p. 1-40, 2002.

${ }^{4}$ BERGER, S. E.; THEURING, C.; ADOLPH, K. E. How and when infants learn to climb stairs. Infant Behavior and Development, v. 30, n. 1, p. 36-49, feb. 2007.

${ }^{5}$ DAVIS, B. E. et al. Effects of sleep position on infant motor development. Pediatrics, Evanston, v. 102, n. 5, p. 1135-1140, nov. 1998.

${ }^{6}$ NORDBERG, L.; RYDELIUS，P.; ZETTERSTRÖM， R. Psychomotor and mental development from birth to age of four years; sex differences and their relation to home environment children in a new stockholm suburb results from a longitudinal prospective study starting at the beginning of pregnancy. Acta Paediatrica, Oslo, v. 80, n. s378, p. 125 , oct. 1991.

${ }^{7}$ LEITSCHUH, C. A.; DUNN, J. M. Prediction of the gross motor development quotient in young children prenatally exposed to cocaine/polydrugs. Adapted Physical Activity Quarterly, v. 18, n. 3, p. 240, july 2001.

${ }^{8}$ LEJARRAGA, H. et al. Psychomotor development in Argentinean children aged 0-5 years. Paediatric and Perinatal Epidemiology, Oxford, v. 16, n. 1, p. 47-60, jan. 2002. 
${ }^{9}$ BAX, M.; WHITMORE, K. The medical examination of children on entry to school. The results and use of neurodevelopmental assessment. Developmental Medicine \& Child Neurology, London, v. 29, n. 1, p. 40-55, feb. 1987.

${ }^{10}$ CAMP, B. W. et al. Preschool developmental testing in prediction of school problems studies of 55 children in Denver. Clinical Pediatrics, Philadelphia, v. 16, n. 3, p. 257-263, mar. 1977.

${ }^{11}$ KROMBHOLZ, H. Physical performance in relation to age sex social class and sports activities in kindergarten and elementary school. Perceptual and Motor Skills, Missoula, v. 84, n. 3c, p. 1168-1170, june 1997.

${ }^{12}$ LARSSON, J. O. et al. Developmental screening at four years of age. Relation to home situation, perinatal stress, development and behaviour. Acta Paediatrica, Oslo, v. 83, n. 1, p. 46-53, jan. 1994.

${ }^{13}$ NOBRE, F. S. S. et al. Análise das oportunidades para o desenvolvimento motor (affordances) em ambientes domésticos no Ceará-Brasil. Revista Brasileira de Crescimento e Desenvolvimento Humano, São Paulo, v. 19, n. 1, p. 9-18, 2009.

${ }^{14}$ OLIVEIRA, S. M. S.; ALMEIDA, C. S.; VALENTINI, N. C. Programa de fisioterapia aplicado no desenvolvimento motor de bebês saudáveis em ambiente familiar. Revista Educação Física/UEM, Maringá, v. 23, n. 1, p. 25-35, 2012.

${ }^{15}$ GIAGAZOGLOU, P. et al. Standardization of the motor scales of the Griffiths Test II on children aged 3 to 6 years in Greece. Child: care, health and development, Oxford, v. 31, n. 3, p. 321-330, may 2005.

${ }^{16}$ HINDLEY, C. B. Stability and change in abilities up to five years: Group trends. Journal of Child Psychology and Psychiatry, Elmsford, v. 6, n. 2, p. 85-99, apr. 1965. 
Recebido em: 23 mar. 2014

Aceito em: 20 ago. 2015

Contato: lariwagner@hotmail.com 